\title{
TIME-RESOLVED SPECTROSCOPY OF Q CYG
}

\author{
S. Kafka, ${ }^{1}$ C. Tappert,${ }^{2}$ R. K. Honeycutt, ${ }^{1}$ and A. Bianchini ${ }^{3}$
}

A time-resolved optical spectroscopic study of the long-period $(P=10.08 h)$ old nova $Q$ Cyg has revealed persistent yet erratic absorption features indicating the presence of a wind, while the system was rising to one of its stunded outbursts. Data taken with the WIYN $3.5 \mathrm{~m}$ telescope show P Cygni profiles in the He I triplet lines at $5876 \AA$ and $7065 \AA$, which are absent in the He I singlet emission line at $6678 \AA$, despite the fact that the relevant energy levels of those three lines are at very similar excitation levels. The $\mathrm{H} \alpha$ line often has a redward emission tail, characteristic of a receding outflow, and it possess a blueward absorption profile during only one wind event. Kafka et al. (2003) argued that this behavior is due to the strongly metastable $2{ }^{3} \mathrm{~S}$ effective ground state of the He I triplets, which becomes overpopulated in conditions of low density and a dilute radiation field, orer-populating in turn the $2^{3} \mathrm{P}$ common lower level of He I $5876 \AA$ and $7065 \AA$, leading to absorption from. a low density wind. Therefore, the triplet/singlet lines trace two different parts of the system: the wind and the disk respectively.

Fig 1 shows the strength of the blue-shifted $P$ Cyg absorption in the HeI $5876 \AA$ line vs time, alongside a similar plot of the strength of the red-shifted $\mathrm{H} \alpha$ emission wing, likely to be due to the receding backside of the wind. The strength was calculated as the average intensity, relative to the continuum, over the ranges $\lambda \lambda 5853-5868$ and $\lambda \lambda 6590-6610$, respectively (corrected for orbital motion); it is suggestive that the $\mathrm{H} \alpha$ feature trails the $\mathrm{P}$ Cyg absorption by about an hour. This is modestly supported by the system's behavior on all nights, although the wind events are somewhat weaker on other nights, and have incomplete coverage. Note that the measured red wing of $\mathrm{H} \alpha$ is at a mean recessional velocity of $\sim+1700 \mathrm{~km} \mathrm{sec}^{-1}$, while the measured absorption in He I has a mean blueshift of $\sim-800 \mathrm{~km} \mathrm{sec}^{-1}$. This difference might account for some of the lack of coincidence.

\footnotetext{
${ }^{1}$ Astronomy Department, Indiana University, 319 Swain Hall West, Bloomington, IN 47405, USA.

${ }^{2}$ Departamento de Física, Grupo de Astronomía, Universidad de Concepción, Casilla 160-C, Concepción, Chile.

${ }^{3}$ Dipartimento di Astronomia, Università di Padova, Vicolo dell'Osservatorio 2, I-35122 Padova, Italy.
}

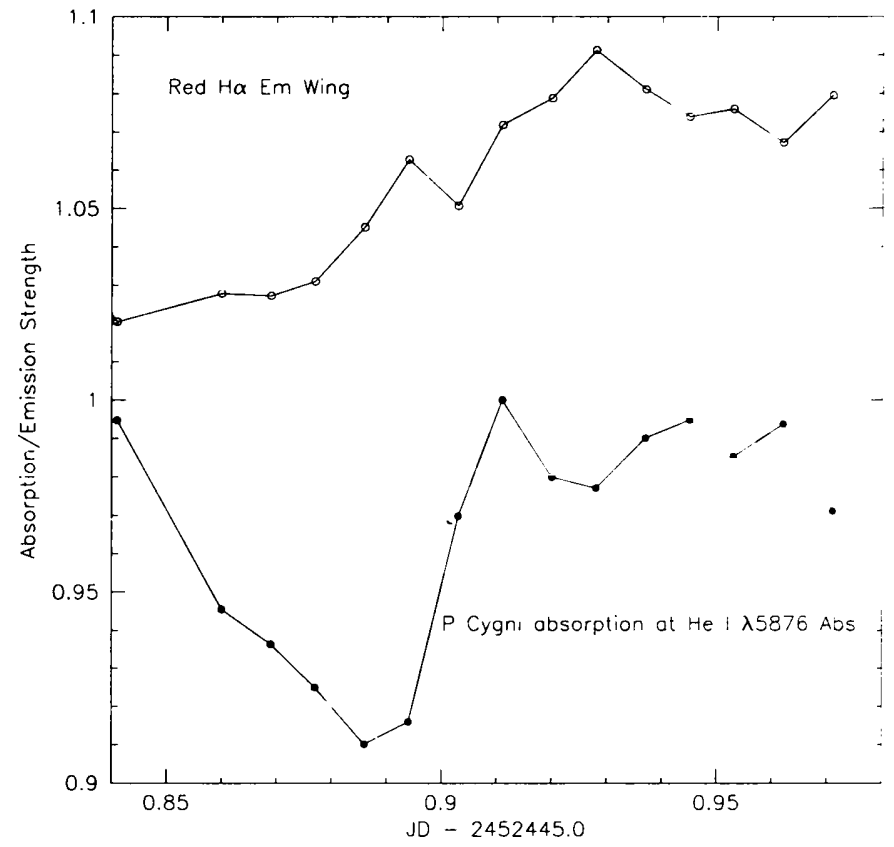

Fig. 1. Strength of the $\mathrm{P}$ Cygni absorption and the strength of the red wing of $\mathrm{H} \alpha$ vs time.

In an attempt to locate the source of the wind. we note the strong orbital modulation of the He I $5876 \AA$ and $7065 \AA$ emission lines and the fact that their $\mathrm{EW}$ is almost zero when the secondary is near inferior conjunction, suggesting a wind origin that is almost totally eclipsed by the secondary star despite the low inclination of the system (also see Kafka et al. 2003). On the other hand, the P Cygni absorption is not orbitally modulated. WIth the data in hand we cannot reach definite conclusions on the mechanism and the location of the wind. However. $\mathrm{Q}$ Cyg appears to have reliable wind lines that are easily accessible in the optical, a promising situation for future studies of this phenomenon.

\section{REFERENCES}

Kafka, S., Tappert, C., Honeycutt, R. K.. \& Bianchini. A. 2003, AJ, 126, 1472

Cannizzo, J. K. \& Cameron, A. G. II 198s. ApJ. 330. 327

Proga, D., Stone, J. M., \& Drew, J. E. 1998, MNRAS. 295,595

Shlosman, I. \& Vitello, P. 1993, ApJ, 409, 372 